\title{
Microalgae as Alternative Sources of Poly-unsaturated Fatty Acids and Antioxidants for Cosmetic Use \\ Gam Da Hye $^{1}$, Song Yi Kim ${ }^{1}$, Ji Woo Hong ${ }^{1}$, Jun Hee Lee ${ }^{1}$, Sayaka Imamura ${ }^{1}$ and Jin Woo Kim ${ }^{1,2^{\star}}$
}

${ }^{1}$ Department of Food Science, Sunmoon University, Asan city, Chung Nam, 380-701, South Korea

${ }^{2}$ FlexPro biotechnology, Start-up Incubation Center, 309, Asan city, Chung Nam, 380-701, South Korea

\section{Abstract}

A growing need for alternatives to replace synthetic cosmetic ingredients and sustainable production process led to interest in natural sources. Among the traditional sources, marine organisms, especially microalgae have shown considerable benefits in terms of land use, waste disposal, and bioactive compound contents. Skin aging is directly related to ultraviolet (UV) radiation which stimulates matrix metalloproteinase (MMP) expression. Continued exposure and increased age also correspond to greater oxidative stress. Fatty acids, especially poly-unsaturated fatty acids (PUFAs) and antioxidants are major materials used in cosmetics to supplement skin aging defensive bioactive materials that are not synthesized in the human body or deficient. Microalgae are suitable alternatives not only for their eco-friendly cultivation process but also for the rich content of omega-3, omega-6, vitamin B, folic acid, carotenoids, and various pigments that act as antioxidants.

\section{Introduction}

The media and society have shown increased interest in skincare, leading to increased production of cosmetics. The focus has mainly shifted to aging, and industries are seeking various sources to a functional ingredient of whitening and anti-aging. To find solutions to whitening and skin aging, synthetics have been developed to be used as ingredients. In recent years, environmental awareness and the demand for natural sources brought greater interest to plants, terrestrial, and marine organisms. Among the various biomass, microalgae are receiving much attention as it is known to have a high effect on carbon dioxide fixing along with fast growth rates and to be able to produce various bioactive substances including polyunsaturated fatty acids and antioxidants for cosmetics.

Microalgae is a photosynthetic organism that can grow in a variety of environments, including fresh water, sea water, and even wastewater [1]. They are highly adaptable and can withstand a wide range of temperature, salinity, $\mathrm{pH}$ values, light intensity, and carbon dioxide concentration. Also, they can grow alone as well as through symbiosis with other organisms [2]. Algae are generally classified as red algae (Rhodophyta), brown algae (Phaeophyta) and green algae (Chlorophyta). Depending on the physical size, algae can be classified into macroalgae and microalgae. Macroalgae, also known as seaweed, are multicellular algae that can be observed with the naked eye, and are used for food or as alternative protein sources. While microalgae are unicellular species, either prokaryotic or eukaryotic, which are significantly smaller in size which are shown through microscopic observation [3,4]. Interest in discovering alternative sources of bioactive materials based on species diversity, incubation convenience and gene manipulation has recently shifted from macroalgae to microalgae, because of the abundance of bioactive molecules such as fatty acids, chlorophyll, vitamins, bioactive compounds and antioxidants which are widely used in cosmetics and food materials.

Domesticating animals and plants require a large amount of arable land and while they also cause severe pollution which can affect human health. When compared to terrestrial plant species, microalgae produce over 10 times more types of beneficial compounds including metabolites [5]. The process of cultivation is sustainable as the carbon dioxide biofixation capability helps absorb emissions. Interest in microorganisms as renewable and sustainable raw materials to produce high value-added bioactive materials has focused new attention on microalgae culture. Growth enhancement techniques and genetic engineering can be used to commercialize the potential of bioactive compounds from microalgae as high value-added materials of the future. Microalgae have been used throughout history for their nutritional benefits. Nostoc, is a genus of cyanobacteria found in various environment, was consumed as an alternative food source during times of famine in China and Aztecs collected masses of Spirulina and Spirulina [6]. The industrial cultivation of microalgae to produce bioactive materials for cosmetics, functional food, and pharmaceuticals has increased dramatically over the last few decades. Microalgae are produced in quantity and sold directly as food and nutrient supplements, while their processed products or extracts are used in cosmetics and biopharmaceuticals [7]. However, the advantages of microalgae consumption have been vaguely known for decades, their effects on the human skin and application still remain relatively unexplored. This study reviews the antioxidant effect of microalgae products and their applications in cosmetics industries for anti-aging.

\section{Algal Anti-aging Substrates}

Extrinsic and intrinsic aging both lead to commonly characterized features of aged skin such as wrinkling, laxity, and sagging. Extrinsic aging, or photoaging, is caused by regular ultraviolet (UV) light exposure. This is the direct cause of connective tissue alterations and other structural changes which are associated with pigmentation and telangiectasia [8,9]. Intrinsic skin aging refers to aging caused largely by genetic factors. Both extrinsic and intrinsic aging are highly tied to collagen degradation. Collagen is the main structural component of the human skin and the decrease or degradation of collagen is considered a major cause of skin aging. Collagen degradation is related to matrix ${ }^{*}$ Corresponding Author: Dr. Jin Woo Kim, Department of Food Science, Sunmoon University, Asan city, Chung Nam, 380-701, South Korea; E-mail: kimjw1028@sunmoon.ac.kr

Citation: Hye GD, Kim SY, Hong JW, Lee JH, Imamura S, et al. (2020) Microalgae as Alternative Sources of Poly-unsaturated Fatty Acids and Antioxidants for Cosmetic Use. Int J Clin Nutr Diet 6: 150. doi: https://doi.org/10.15344/2456$8171 / 2020 / 150$

Copyright: (c) 2020 Hye et al. This is an open-access article distributed under the terms of the Creative Commons Attribution License, which permits unrestricted use, distribution, and reproduction in any medium, provided the original author and source are credited. 
metalloproteinases (MMPs). MMP levels show increased expression as a response to UV radiation, oxidative stress, and other stimuli [10]. Increasing age is another factor that could stimulate MMP expression. Fatty acids are essential components of the skin both structurally and functionally. As photosynthetic primary producers, microalgae produce fatty acids while using carbon dioxide.

The three types of omega-3 fatty acids involved in human physiology are $\alpha$-linolenic acid (ALA), found in plant oils, and eicosapentaenoic acid (EPA) and docosahexaenoic acid (DHA), both commonly found in marine oils. According to previous studies, omega-3 and omega- 6 cannot be synthesized by the human body therefore a variety of traditional protein sources were used as omega-3 supplements. Marine algae and phytoplankton are primary sources ofomega-3 fatty acids and microalgae rich in omega- 3 is recognized as a good source of nutrition for humans, especially the elderly and children. Studies show that when compared to these commonly used sources of polyunsaturated fatty acids (PUFA), microalgae are richer or comparable in EPA and DHA content. In the case of Chlorella minutissima and Nannochloropsis salina, they showed 39.9\%EPA and 28\%EPA when compared to soybean and Oncorhynchus gorbuscha which had $20.0 \% \mathrm{EPA}$ and 27.5\%EPA+DHA respectively [11]. Among the lipid component of microalgae, the fatty acid portion ranges from $25-60 \%$, depending on the species [12].

The skin's outermost layer, or epidermis, is mainly consisted of natural lipids which are made up of fatty acids [13]. Omega- 3 and omega- 6 are two essential fatty acids determined to be unsaturated. Omega-3 broadly includes eicosapentaenoic acid, docosahexaenoic acid, and $\alpha$-linolenic acid [14]. EPA is known for its inflammatory benefits as well as inhibiting MMP expressions induced by UV radiation [15]. Reports on Omega-3 fish oil supplementations and their MMP activity inhibition and collagen synthesis effects [16]. Microalgae is also known to produce and accumulation of large amount of lipid, including glycerol and other types of fatty acids (1222 carbons) which acids are highly nutritious and functional, and are used as food and cosmetic substrate [17]. Extracts from microalgae have various bioactive substances which accelerate the healing process and maintain skin moisture. In particular, the moisturizing effect and skin regeneration effect of microalgae fatty acid have been proven, making it a popular ingredient for cosmetics [18]. Studies were conducted at the gene level to determine the cause of anti-aging and skin regeneration. They may involve the anti-aging gene (Sirtuin 1) that when activated may maintain skin function and anti-aging properties. The microalgae may contain anti-aging factors such as Sirtuin 1 activators that are critical to maintain DNA repair systems and skin function. Sirtuin 1 is critical for skin regeneration and Sirtuin 1 activators found in microalgae may be the critical components to maintain skin regeneration and health [19-22].

\section{Algal Anti-oxidant Substrates}

Oxidative stress is the imbalance between the levels of reactive oxygen species (ROS) and antioxidants, which results in the accumulation of ROS and consequently contributes greatly to aging. Oxidative can occur in various essential components of the human body such as proteins and DNA. In terms of extrinsic aging, exposure to UV radiation leads to photochemical generation of ROS as the radiation inactivates DNA by yielding photoproducts. Further damage can be done including sister chromatid exchange, stand breaks, mutations and more [23]. ROS levels also increase as a result of intrinsic aging. An increase in age corresponds to the decreased ability of cells to repair DNA damage caused by accumulated ROS. Energy is needed in the repair process which is generated through processes such as mitochondrial oxidative phosphorylation. As the body ages, mitochondria are functionally less productive and thus produces less energy. In response to such oxidative stress, the human body has an antioxidative defense mechanism which restores the balance. Different forms of antioxidants exist, such as uric acid, catalase, vitamins, carotenoids and phenolic compounds [24]. While endogenous antioxidants are present in the skin, their concentrations decrease as aging proceeds or the skin is exposed to stressors. Therefore, topical application or consumption of these bioactive materials through food is needed to complement the deficiency of antioxidants. Microalgae are also rich in antioxidants. Vitamins that can be found in microalgae include vitamin $B_{1}, B_{12}, A, C, E$, and folic acid [25].

The photosynthetic nature of microalgae is what produces a variety of natural pigments such as chlorophyll, phycobiliproteins, and carotenoids in comparably high concentrations [26]. These pigments not only give the color of the algae by absorbing specific wavelengths but also act as antioxidants. The porphyrin ring structure of chlorophyll a is responsible for its antioxidant activity as it reacts with peroxyl radicals $\left(\mathrm{RO}_{2} \bullet\right)$ to inactivate them [27]. Microalgae pigments are also in the spotlight as cosmetic products due to their high anti-oxidization activities, which are known to be effective in preventing melanin pigment production and skin aging through the inhibiting of tyrosinase activity, a major enzyme in the production of melanin pigments, and the inhibiting of collagenase active, which causes skin aging. Fucoxanthin, a pigment that gives a brownish tint found in Hijikia fusiformis, showed high radical scavenging activity and cytoprotective effect which leads to ROS suppression [28].Blue-green microalgae (cyanobacteria) is produced by various photosynthetic pigments (carotenoids) that have fast growth and antioxidant production due to their high efficiency in the use of light energy. Currently, microalgae pigments, such as carotenoids, are used as additives to various foods such as candy bars, gums, pasta and beverages [29].

\section{Conclusions}

Microalgae is an effective small plant that uses solar energy and produces useful materials by fixing carbon dioxide and is a renewable, sustainable and economical source of bioactive ingredients. Among them, polysaccharides, carotene, lipids, and proteins are known to have numerous positive effects on health. As the need for natural antioxidants and fatty acid sources as alternatives to synthetics, many microalgae are used to produce various bioactive materials and have gained interest within the cosmetics industry. These cases show how microalgae extracts can be applied for anti-aging and antioxidant skin care products for their rich composition of bioactive materials. Many microalgae strains are cultivated on a commercial scale for their high bioactive materials and other health-promoting substances in the area of the cosmetics, food and medicine industries [30]. Many studies have been conducted on the functionality of materials produced by microalgae. Also, studies have been actively conducted to identify ingredients from microalgae as new research areas and to reveal biological and medical functions. Microorganisms are useful in mitigating $\mathrm{CO}_{2}$ fixation, biomass production and wastewater treatment, and can be expanded from pilot scale to commercial level by overcoming challenges and limitations on mass production. Such mass industrialization will lead to the production and popularization of more price-competitive micro-algae cosmetics [31]. As a relatively 
Citation: Hye GD, Kim SY, Hong JW, Lee JH, Imamura S, et al. (2020) Microalgae as Alternative Sources of Poly-unsaturated Fatty Acids and Antioxidants for Cosmetic Use. Int J Clin Nutr Diet 6: 150. doi: https://doi.org/10.15344/2456-8171/2020/150

Page 3 of 3

unknown source of antioxidants and fatty acids, further research can be carried on to investigate the suitable methods of extracting these compounds to maximize and optimize the yield according to extraction conditions.

In conclusion, with increasing demand for natural products for skin care and well-being treatments, microalgae can be an important source of material with bioactive effects on skin health. Microalgae, which includes abundant antioxidants and skin regeneration materials, is believed to be an excellent cosmetic ingredient with UV protection and ability to regenerate skin cells. Therefore, research and commercialization of microalgae, which is capable of effective production of various bioactive materials of microalgae, will certainly continue to expand.

\section{Competing Interests}

The authors declare that they have no competing interests.

\section{Author Contributions}

JWK and DHG are responsible for conception and design of the review. All authors contributed to analysis of the literature, writing, and critical reflecting of the manuscript. All authors have seen and approved the final version of the manuscript.

\section{References}

1. Yan C, Fan J, Xu C (2013) Analysis of oil droplets in microalgae. Methods Cell Biol 116: 71-82.

2. Barsanti L, Coltelli P, Evangelista V, Frassanito AM, Passarelli V, et al. (2008) Oddities and curiosities in the algal world. Algal toxins: nature, occurrence effect and detection.

3. Khan MI, Shin JH, Kim JD (2018) The promising future of microalgae: current status, challenges, and optimization of a sustainable and renewable industry for biofuels, feed, and other products. Microb Cell Fact 17: 36

4. AbdEl-Hack ME, Abdelnour S, Alagawany $M$, Abdo $M$, Sakr MA, et al. (2018) Microalgae in modern cancer therapy: Current knowledge. Biomed Pharmacother 111: 42-50.

5. Fu W, Nelson DR, Yi z, Xu M, Khraiwesh B, et al. (2017) Bioactive Compounds from Microalgae: Current Development and Prospects. Studies in natura products chemistry 54: 199-225

6. Garcia JL, Vicente MF, Galan BG (2017) Microalgae, old sustainable food and fashion nutraceuticals. Microb Biotechnol 10: 1017-1024.

7. Luiten EE, Akkerman I, Koulman A, Kamermans P, Reith $\mathrm{H}$, et al. (2003) Realizing the promises of marine biotechnology. Biomol Eng 20: 429-439.

8. Kim EJ, Kim MK, Jin XJ, Oh JH, Kim JN, et al. (2010) Skin aging and photoaging alter fatty acids composition including 11,14,17-eicosatrienoic acid, in the epidermis of human skin. J Korean med Sci 25: 980-983.

9. Kim HH, Cho SY, Lee SA, Kim KH, Eun HC, et al. (2006) Photoprotective and anti-skin-aging effects of eicosapentaenoic acid in human skin in vivo. Lipid Res 47: 921-930.

10. Pittayapruek $P$, Meephansan J, Prapapan $O$, Kominee $M$, Ohtsuki $M$ et al. (2016) Role of matrix metalloproteinases in photoaging and photocarcinogenesis. Int JMol Sci 17: 868.

11. Adarme-Vega TC, Lim DK, Timmins M, Vernen F, Li Y, et al. (2012) Microalgal biofactories: a promising approach towards sustainable omega-3 fatty acid production. Microb Cell Fact 11: 96.

12. Richmond A (2004) Handbook of Microalgal Culture: Biotechnology and Applied Phycology, England.

13. Hansen HS, Jensen B (1985) Essential function of linoleic acid esterified in acylglucosylceramide and acylceramide in maintaining the epidermal water permeability barrier. Evidence from feeding studies with oleate, linoleate, arachidonate, columbinate and alpha-linolenate. Biochim Biophys Acta 834 357-363.
14. Mirnikjoo B, BrownSE, KimHF, MarangellL B, SweattJ D, et al. (2001) Protein kinase inhibition by omega-3 fatty acids. J Biol Chem 276: 10888-10896.

15. Kim HH, Shin $\mathrm{CM}$, Park $\mathrm{CH}_{1} \mathrm{Kim} \mathrm{KH}$, Cho KH, et al. (2005) Eicosapentaenoic acid inhibits UV-induced MMP-1 expression in human dermal fibroblasts. J Lipid Res 46: 1712-1720.

16. Poljsak B, Dahmane R, Godic A (2012) Intrinsic skin aging: The role of oxidative stress. Acta dermatovenerologica Alpina 21:33-36.

17. Duong VT, Ahmed F, Thomas-Hall SR, Quigley S, Nowak E, et al. (2015) High protein- and high lipid-producing microalgae from northern australia as potential feedstock for animal feed and biodiesel. Front Bioeng Biotechnol 3: 53.

18. Martins IJ (2018) Anti-Aging Gene linked to Appetite Regulation Determines Longevity in Humans and Animals. Int J Aging Res 1: 1-4.

19. Talero E, García-Mauriño S, Ávila-Román J, Rodríguez-Luna A, Alcaide A, et al. Bioactive Compounds Isolated from Microalgae in Chronic Inflammation and Cancer. Mar Drugs 13: 6152-209.

20. Garcia-Peterson LM, Wilking-Busch MJ, Ndiaye MA, Philippe CGA, Setaluri $\mathrm{V}$, et al. (2017) Sirtuins in Skin and Skin Cancers. Skin Pharmacol Physiol 30: 216-224.

21. Martins IJ (2017) Nutrition Therapy Regulates Caffeine Metabolism with Relevance to NAFLD and Induction of Type 3 Diabetes. J Diabetes Metab Disord 4: 019.

22. Kim SK, Ravichandran YD, Khan SB, Kim YT (2008) Prospective of the cosmeceuticals derived from marine organisms. Biotechnol Bioproc Eng 13: 511-523.

23. Poljsak B, Dahmane R (2012) Free radicals and extrinsic skin aging. Dermatol Res Pract 12: 135-206.

24. Schagen SK, Zampeli VA, Makrantonaki E, Zouboulis CC (2012) Discovering the link between nutrition and skin aging. Dermatoendocrinol 4: 298-307.

25. Spolaore $P$, Joannis-Cassan C, Duran E, Isambert A (2006) Commercial applications of microalgae. J Biosci Bioeng 101: 87-96.

26. Koyande AK, Chew KW, Rambabu K, Tao Y, Chu DT, et al. (2019) Microalgae: A potential alternative to health supplementation for humans. Food $\mathrm{Sci}$ Human Wellness 8: 16-24.

27. Pangestuti R, Kim SK (2011) Biological activities and health benefit effects of natural pigments derived from marine algae. J Funct Foods 3: 255-266.

28. Sachindra N, Sato E, Maeda H, Hosokawa M, Niwano Y, et al. (2007) Radical scavenging and singlet oxygen quenching activity of marine carotenoid fucoxanthin and its metabolites. J Agric Food Chem 55: 8516-8522.

29. Soletto D, Binaghi L, Lodi A, Carvalho JCM, Converti A, et al. (2005) Batch and fed-batch cultivations of Spirulina platensis using ammonium sulphate and urea as nitrogen sources. Aquaculture 243: 217-224.

30. Liang S, Xueming L, Chen F, Chen Z (2004) Current microalgal health food R\&D activities in China. Hydrobiologia 512: 45-48.

31. Pulz O (2001) Photobioreactors: production systems for phototrophic microorganisms. Appl Microbiol Biotechnol 57: 287-293. 